\title{
A systematic review of the role of vitamin $D$ and calcium in premenstrual syndrome
}

\author{
Fatemeh Abdi', Gity Ozgoli², Fatemeh Sadat Rahnemaie ${ }^{1}$ \\ ${ }^{1}$ Student Research Committee, ${ }^{2}$ Department of Midwifery, Nursing and Midwifery Faculty, Shahid Beheshti University of Medical Sciences, Tehran, Iran
}

Premenstrual syndrome (PMS) is a common disorder that affects millions of women of reproductive age worldwide. In recent years, there has been a focus on finding accessible, acceptable, and cost-effective therapeutic approaches with minimal side effects to treat the symptoms of PMS. This systematic review aimed to investigate the role of calcium and vitamin D in Premenstrual syndrome. The PubMed, EMBASE, Web of Science, Scopus, Science Direct, and Google Scholar databases were systematically searched for relevant articles from clinical trial, case-control, and crosssectional studies. The Strengthening the Reporting of Observational Studies in Epidemiology checklist was used to assess the quality of the selected papers. A total of 28 eligible high-quality papers were reviewed. Low serum levels of calcium and vitamin D during the luteal phase of the menstrual cycle were found to cause or exacerbate the symptoms of PMS. Therefore, the administration of calcium and vitamin D supplements or the use of a diet rich in these two substances can restore serum levels and eliminate or reduce the symptoms of PMS. Calcium and vitamin D supplementation are recommended as an inexpensive, low-risk, acceptable, and accessible approach to eliminate or reduce the symptoms of PMS.

Keywords: Premenstrual syndrome; Vitamin D; Calcium

\section{Introduction}

Premenstrual syndrome (PMS) is a common disorder that affects millions of women of reproductive age worldwide [1]. PMS is a series of physical symptoms (e.g., edema, weight gain, mastalgia, headache, nasal obstruction, and paresthesia) and psychological complaints (e.g., irritability, anxiety, and depression) experienced by most women during the luteal phase of the menstrual cycle (7-14 days before the menstrual period) $[2,3]$. The symptoms of PMS can vary from person to person and, since physical, emotional, and behavioral symptoms may have different causes, the biological factors and symptoms of PMS should be separately evaluated [4]. The incidence of PMS depends on the sociocultural conditions and characteristics of the studied women and the diagnostic methods applied. However, the prevalence of PMS has been estimated to be 30-40\% among women of reproductive age and 20-32\% among premenopausal women [5].

The main cause of PMS is still unknown, and the syndrome is most likely multifactorial. Some researchers argue that hormonal agents and a lack of micronutrients might be involved in the development of PMS [6]. In consideration of the role that various factors can play in PMS, several therapeutic approaches have been proposed for symptom control that include the use of medications, particularly supplements and anti-depressants, dietary modifications, psychological approaches, exercise, and relaxation methods (e.g., yoga). However, researchers are always searching for more efficient, cost-effective, and safer therapies [7]. The World Health Organization and legislative institutions are also interested in the identification of accessible and inexpensive treatments

Received: 2018.08.20. Revised: 2018.09.27. Accepted: 2018.11.04. Corresponding author: Fatemeh Sadat Rahnemaie Student Research Committee, Nursing and Midwifery Faculty, Shahid Beheshti University of Medical Sciences, Chamran Highway - Yaman Street - Shahid Arabi Street - Beside Ayatollah Taleghani Hospital, Tehran 19839-63113, Iran

E-mail: F_rahnemaie@yahoo.com

https://orcid.org/0000-0002-1149-8057

Articles published in Obstet Gynecol Sci are open-access, distributed under the terms of the Creative Commons Attribution Non-Commercial License (http://creativecommons. org/licenses/by-nc/3.0/) which permits unrestricted non-commercial use, distribution, and reproduction in any medium, provided the original work is properly cited.

Copyright $\odot 2019$ Korean Society of Obstetrics and Gynecology 


\title{
Obstetrics \& Gynecology Science
}

\author{
Vol. 62, No. 2, 2019
}

with minimal side effects to control PMS symptoms [8]. According to previous research, $80 \%$ of women suffering from PMS seek complementary and alternative therapies [9], and vitamin $D$ and calcium have recently received special attention for the treatment/relief of PMS symptoms.

Vitamin $D$ is a hormone involved in not only calcium and phosphorous metabolism, but also several other important metabolic functions $[10,11]$. In humans, $80 \%$ of the body's daily requirement of vitamin $D$ is usually fulfilled by 7-Dehydrocholesterol on ultraviolet B exposure, and the remaining $20 \%$ is received through dietary intake. The role of vitamin $D$ in reducing the risk of PMS is still being studied and seems primarily correlated with the modulation of calcium concentrations, certain neurotransmitters, and sexual steroids [12].

Vitamin D plays a role in the differentiation and proliferation of various cells [13]. Some previous studies reported changes in serum 25-hydroxyvitamin D3 ([25(OH)D3] or $[25(\mathrm{OH}) \mathrm{D}])$ during the luteal phase [12]. Ovarian sex hormones induce enzymes that degrade [25(OH)D3]. Estradiol induces incremental catalytic activity of 1-a-hydroxylase and 24-hydroxylase in the liver, which decrease serum [25(OH)D3] $[14,15]$. Ovarian hormones peak during the luteal phase, and this leads to the degradation of [25(OH)D3] and reduction of its serum levels. As a result, the cyclic disestablishment of serum [25(OH)D3] metabolism by the ovarian hormones could worsen the symptoms of PMS [16]. Vitamin D deficiency is also associated with an increased renin angiotensin aldosterone system function that contributes to increased fluid balance, blood pressure changes, and hypertension $[17,18]$.

Some studies have shown that serum calcium levels increase during different stages of menstrual cycle and are lower during the luteal phase than during the follicular phase [19]. Decreased serum calcium levels have also been reported during the premenstrual period in some women [16]. Differ- ent studies suggest that calcium deficiency during the luteal phase of the menstrual cycle can exacerbate PMS symptoms by causing depression, hallucination, and restlessness [20,21]. Moreover, women who have diets rich in vitamin D and calcium are at a lower risk of developing PMS compared to other women [22].

Systematic reviews explicitly summarize the results of previous studies and provide the best form of evidence for an unbiased judgment. To the best of our knowledge, no systematic review has focused on the effects of vitamin $D$ and calcium in the improvement of PMS symptoms, and therefore this study reviewed the role of vitamin D and calcium in PMS. The results of this study can be helpful in decision-making for the administration of alternative therapies to women who want to take advantage of low-risk treatments.

\section{Criteria for considering studies for this review}

\section{Search strategy}

This study was reported based on the Preferred Reporting Items for Systematic Reviews and Meta-Analyses guidelines. A number of key terms were searched including: 'premenstrual syndrome' OR 'premenstrual dysphoric disorder' OR 'premenstrual tension' OR 'PMS' AND 'vitamin D' OR 'cholecalciferol' OR 'hydroxycholecalciferol' OR 'vitamin D3' OR '25-hydroxyvitamin D3' AND 'Calcium' OR 'Ca'. In order to collect the data, valid databases (i.e., MEDLINE, ISI Web of Science, PubMed, EMBASE, Scopus, Google Scholar, and Science Direct) were systematically searched (Table 1).

\section{Inclusion and exclusion criteria}

Studies were included if they were published in either English

Table 1. Search strategy for PubMed and EMBASE

\begin{tabular}{ll}
\hline Search number & \multicolumn{1}{c}{ Search term } \\
\hline \#1 & $\begin{array}{c}\text { 'premenstrual syndrome' [tiab] OR 'premenstrual dysphoric disorder' [tiab] OR 'premenstrual tension' [tiab] OR 'PMS' } \\
\text { \#2 }\end{array}$ \\
\#3 $\begin{array}{l}\text { 'vitamin D' [tiab] OR 'cholecalciferol' [tiab] OR 'hydroxycholecalciferol' [tiab] OR 'vitamin D3' [tiab] OR } \\
\text { '25-hydroxyvitamin D3' [tiab] } \\
\text { \#1 AND \#2 }\end{array}$ & 'Calcium' [tiab] OR 'Ca' [tiab] \\
\#1 AND \#3 & \\
\#1 AND \#2 AND \#3 & \\
\hline
\end{tabular}




\section{Obstetrics \& Gynecology Science}

Fatemeh Abdi, et al. The role of calcium and vitamin $D$ in premenstrual syndrome

or Persian during 1999-2018 and if they had a case-control, clinical trial, quasi-experimental, or cross-sectional design. Other inclusion criteria were menarche at least one year before the intervention, regular menstrual cycles, moderate to severe PMS symptoms in the second half of the menstrual cycle, no use of hormone therapy or other therapeutic approaches for the treatment of PMS, and no pregnancy.

The exclusion criteria were: chronic diseases; use of calcium and vitamin D supplements; use of other drugs such as antidiabetes and anti-inflammatory drugs during the six-month period before the intervention; mental disorders such as anxiety, depression, and panic attacks; and migraine headaches.

\section{Study selection}

The initial search yielded 1,055 results. The eligibility of these articles was independently evaluated by 2 authors, and any disagreements were resolved through a consensus. During this evaluation, 828 articles were found to be irrelevant or duplicates and were excluded. After reviewing the titles and abstracts of the remaining articles, 173 additional articles were excluded. During the evaluation of the full texts, 26 out of the remaining 54 articles were found ineligible and were excluded. As a result, a total of 28 eligible articles were finally reviewed (Fig. 1).

\section{Quality assessment}

Quality of the quantitative studies was determined by evaluating their adherence to the Strengthening the Reporting of Observational Studies in Epidemiology (STROBE) checklist $[23,24]$. The studies that adhered to all 7 items, 6 items, and 2 or more items of the STROBE checklist were classified as having high, medium, and low-quality, respectively.

\section{Data extraction}

Two authors independently performed the study selection and validity assessment and resolved any disagreements by consulting a third researcher. The first author's name, publication year, country, study design, sample size, chief complaint or problem, intervention group, control group, measures, and outcomes were extracted and entered into the analysis.

\section{Results}

After the initial search, the titles and abstracts of the studies extracted from all the databases were examined. The full texts of the studies that were found eligible during the last review stage were then evaluated, and 28 articles were finally analyzed. All of these studies were eligible based on the inclusion criteria and had high-quality according to their

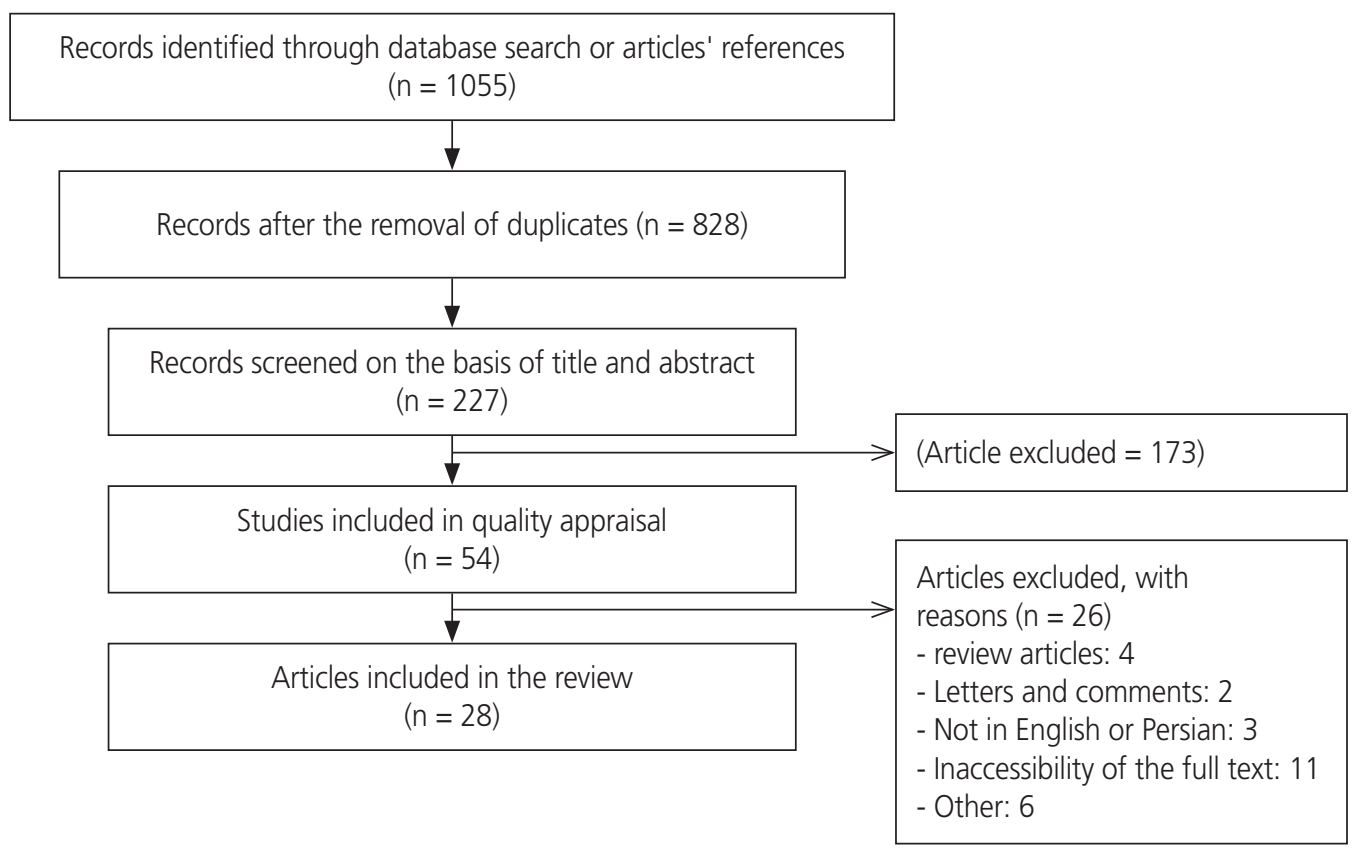

Fig. 1. Search flow diagram. 


\section{Obstetrics \& Gynecology Science}

Vol. 62, No. 2, 2019

adherence to the STROBE checklist. The characteristics of the selected studies are presented in Table 2 . The selected studies had different sample sizes (between 39 and 3,025 women) and examined a total of 8,576 women. The participants of the selected studies were of different ages and from different geographical areas including Iran $(n=15)$, India $(n=4)$, the USA $(n=4)$, Indonesia $(n=1)$, Palestine $(n=1)$, Jordan $(n=1)$, Italy $(n=1)$, and Egypt $(n=1)$. The selected studies used clinical trial $(n=16)$, case-control $(n=7)$, cross-sectional $(n=4)$, and quasi-experimental $(n=1)$ designs. The results of the quality assessment of the quantitative studies based on their adherence to the STROBE checklist are detailed in Table 3.

The results indicated that low serum levels of vitamin $D$ and calcium during the luteal phase of the menstrual cycle could contribute to the incidence and exacerbation of PMS symptoms. Moreover, the mean severity and number of PMS symptoms decreased after calcium and vitamin D supplementation.

\section{Discussion}

This systematic review analyzed the results of high-quality studies regarding the role of calcium and vitamin D in PMS while considering the effects of PMS on the physical and psychological aspects of women's health. The results indicate that low levels of vitamin D and calcium during the luteal phase of menstrual cycle can cause and/or intensify the symptoms of PMS. Calcium and vitamin D supplementation can increase serum levels of these 2 micronutrients and may eliminate or reduce the severity of PMS symptoms. Less severe PMS symptoms were also observed in women using diets rich in vitamin $D$ and calcium.

The positive relationship reported between [25(OH)D3] and PMS symptoms is in contrast to Bertone's hypothesis [49]. Their study showed that [25(OH)D3] levels increased in cases of PMS, due to differences in supplementation methods, and that such results arise from errors in the findings [48]. Although the exact cause of this finding is unclear, the possible use of supplements or multivitamins containing calcium and vitamin D by women with PMS can be one explanation [49].

This finding is consistent with those of Thys-Jacobs et al. [50] who reported insignificantly higher use of calcium supplements (46\% vs. $32 \%$ ) and vitamin D supplements $(41 \%$ vs. $30 \%$ ) among women with premenstrual dysphoric disor- der (PMDD) than among symptom-free controls. The different doses of calcium supplementation during the intervention support the hypothesis that women experiencing PMS may be self-medicating with dietary supplements. Similarly, the associations of vitamin $D$ with specific symptoms may vary. Bertone-Johnson et al. [48] studied women not yet diagnosed with PMS at the time of blood collection and found [25(OH)D3] levels to be associated with a significantly lower risk of developing breast tenderness, diarrhea/constipation, fatigue, and depression $(P<0.05)$ and a possible lower risk of swelling of the extremities and bloating $(P \leq 0.11)$.

In addition, the inverse relationship between [25(OH)D3] levels and PMS confirms previous findings that indicated the possible role of vitamin $D$ in decreasing the risk of unipolar depression [51]. Low serum levels of $\mathrm{Mg}^{++}$and [25(OH)D3] during the luteal phase have also been observed in women suffering from PMS [45]. The findings of the mentioned study are inconsistent with previous research [52-54], and this inconsistency can be justified by differences in the study populations, geographic regions, sample sizes, and sample size estimation methods [45].

Another case-control study analyzed serum $\mathrm{Mg}^{++}$and [25(OH)D3] levels after supplementation in a cohort of 1,057 females and reported less severe PMS symptoms in the case group than in the controls [46]. Serum $\mathrm{Mg}^{++}$and $[25(\mathrm{OH})$ D3] levels are low during the luteal phase of menstrual cycle due to cyclical changes in the release of hormones from the hypothalamic-pituitary-ovarian axis [45]. Calcium supplementation reduced the severity of the mentioned symptoms and changed the frequencies of severe, moderate, and mild symptoms to $7 \%, 33 \%$, and $47 \%$, respectively [40].

A high dose of vitamin D3 (50,000 IU/weekly) is recommended for the prevention and treatment of vitamin $D$ deficiency [55]. Bahrami et al. [25] administered high-dose vitamin D pearls (50,000 IU cholecalciferol/weekly) during a period of 9 weeks and observed a significant relationship between vitamin $D$ therapy and improvement of PMS symptoms and dysmenorrhea among adolescents.

In a placebo-controlled study of the effects of calcium (500 mg) plus vitamin D (200 mg) supplementation on PMS, Khajehei et al. [30] showed that supplementation decreased the severity of PMS symptoms. They compared the mean severity of symptoms between groups before and after the intervention and found that dydrogesterone and calcium plus vitamin $D$ had the same effects on decreasing symptom 


\section{Obstetrics \& Gynecology Science}

Fatemeh Abdi, et al. The role of calcium and vitamin D in premenstrual syndrome

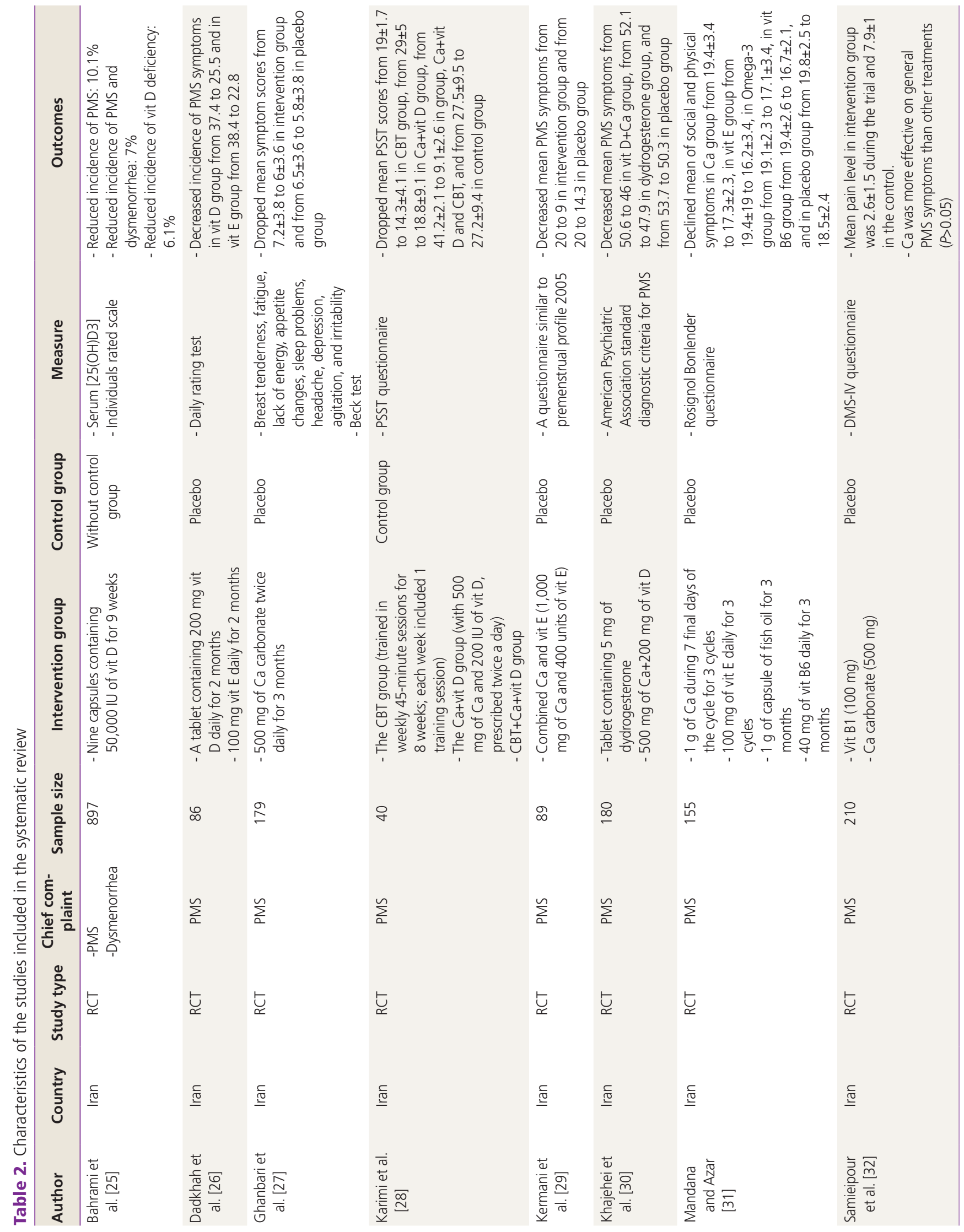




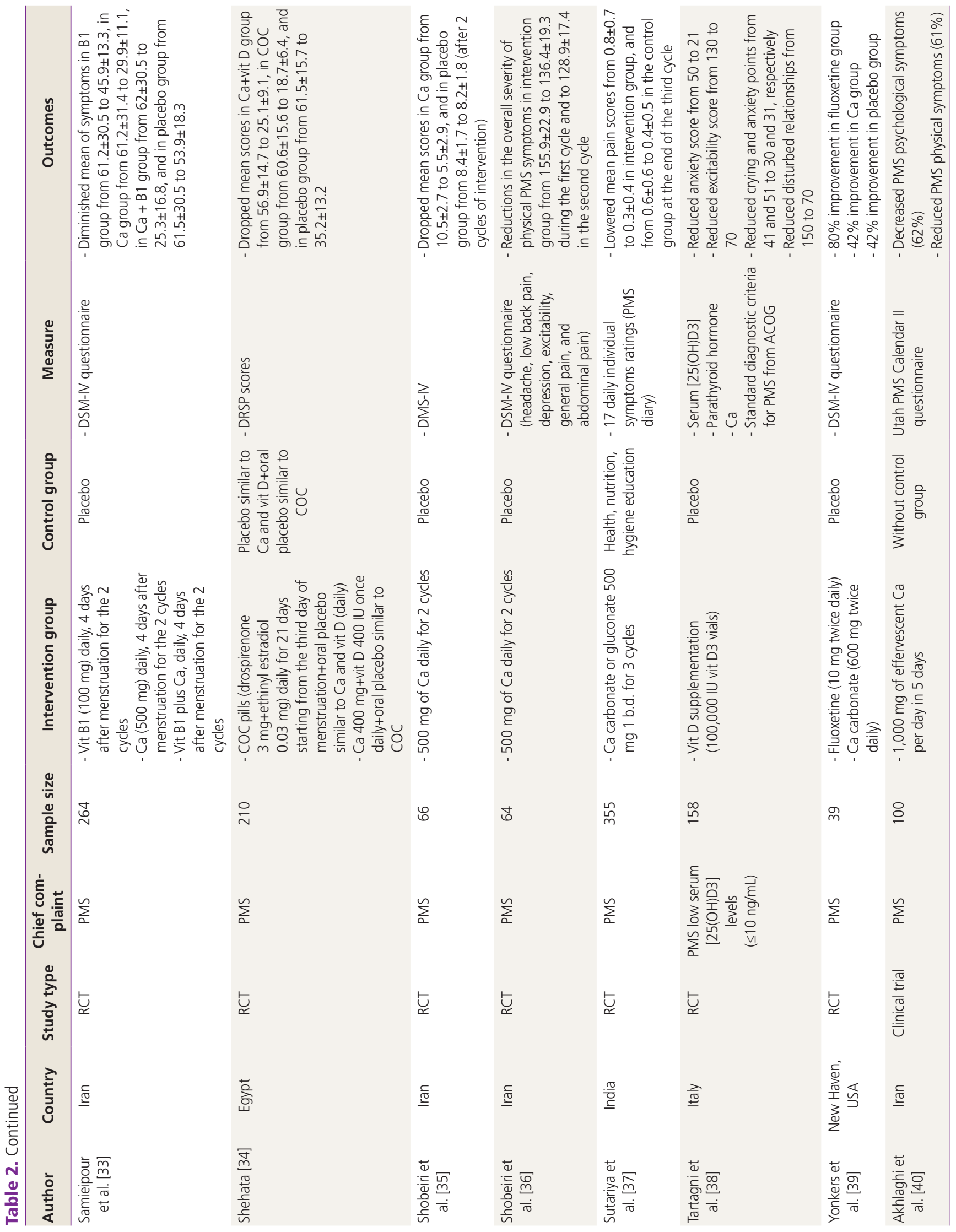




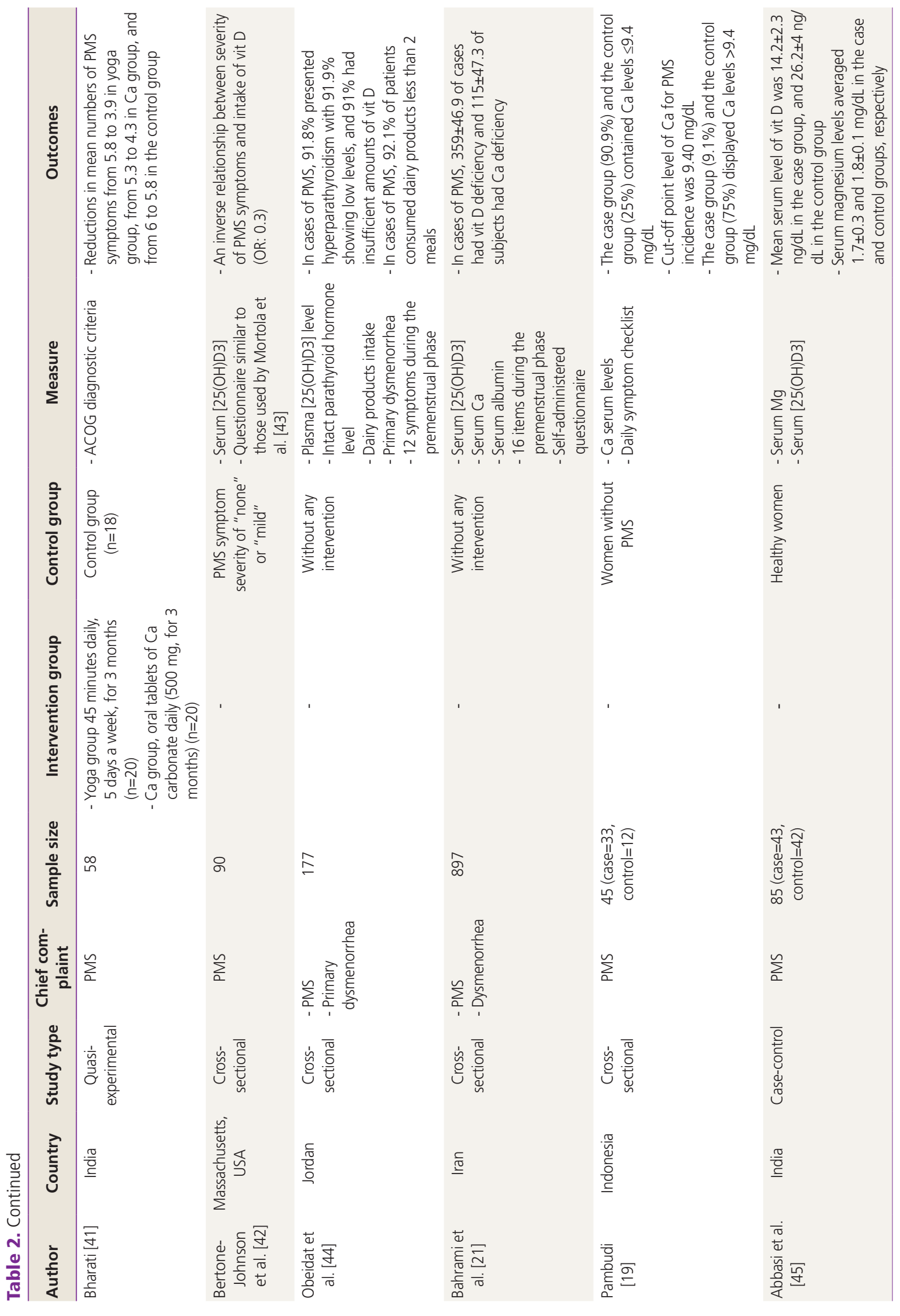




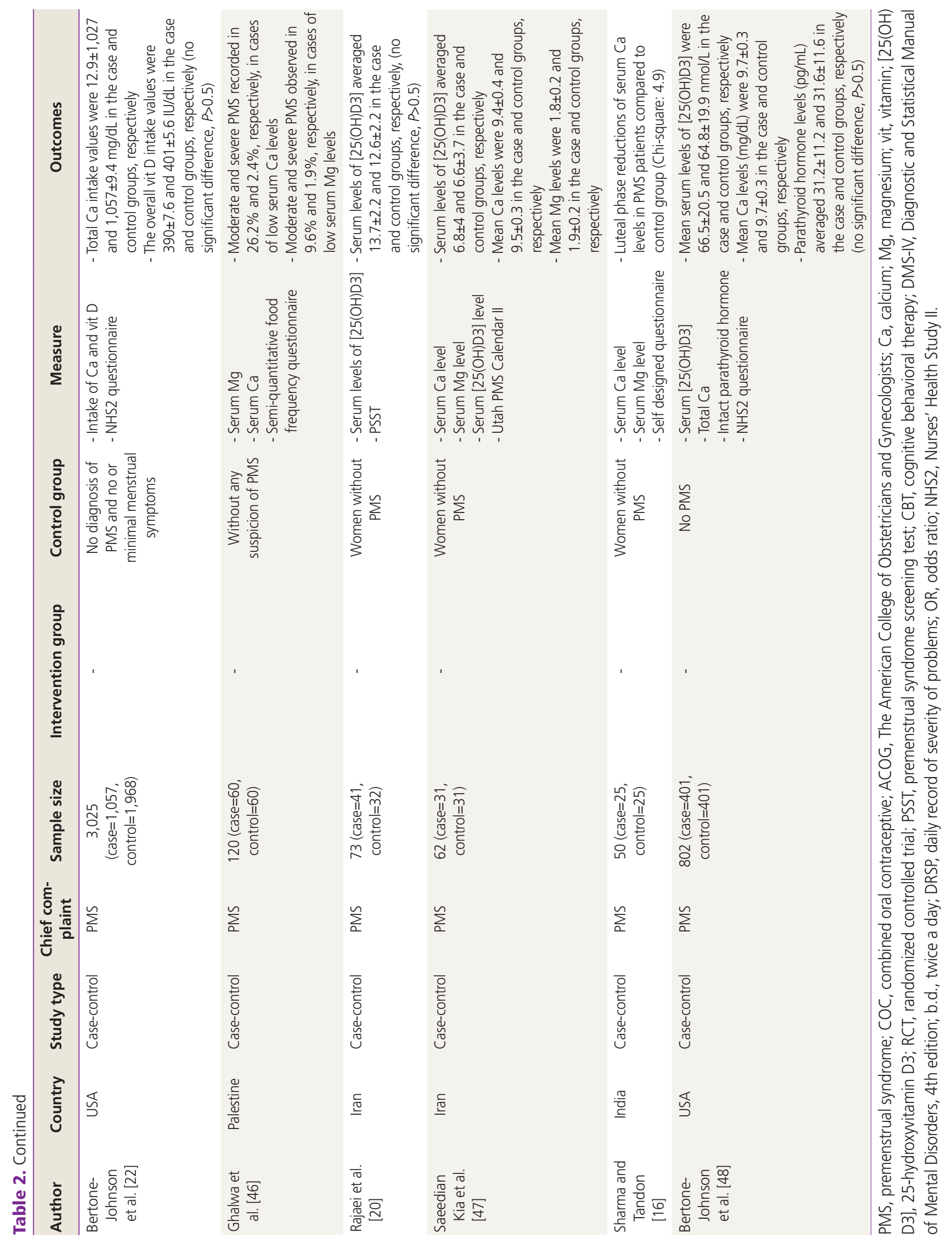




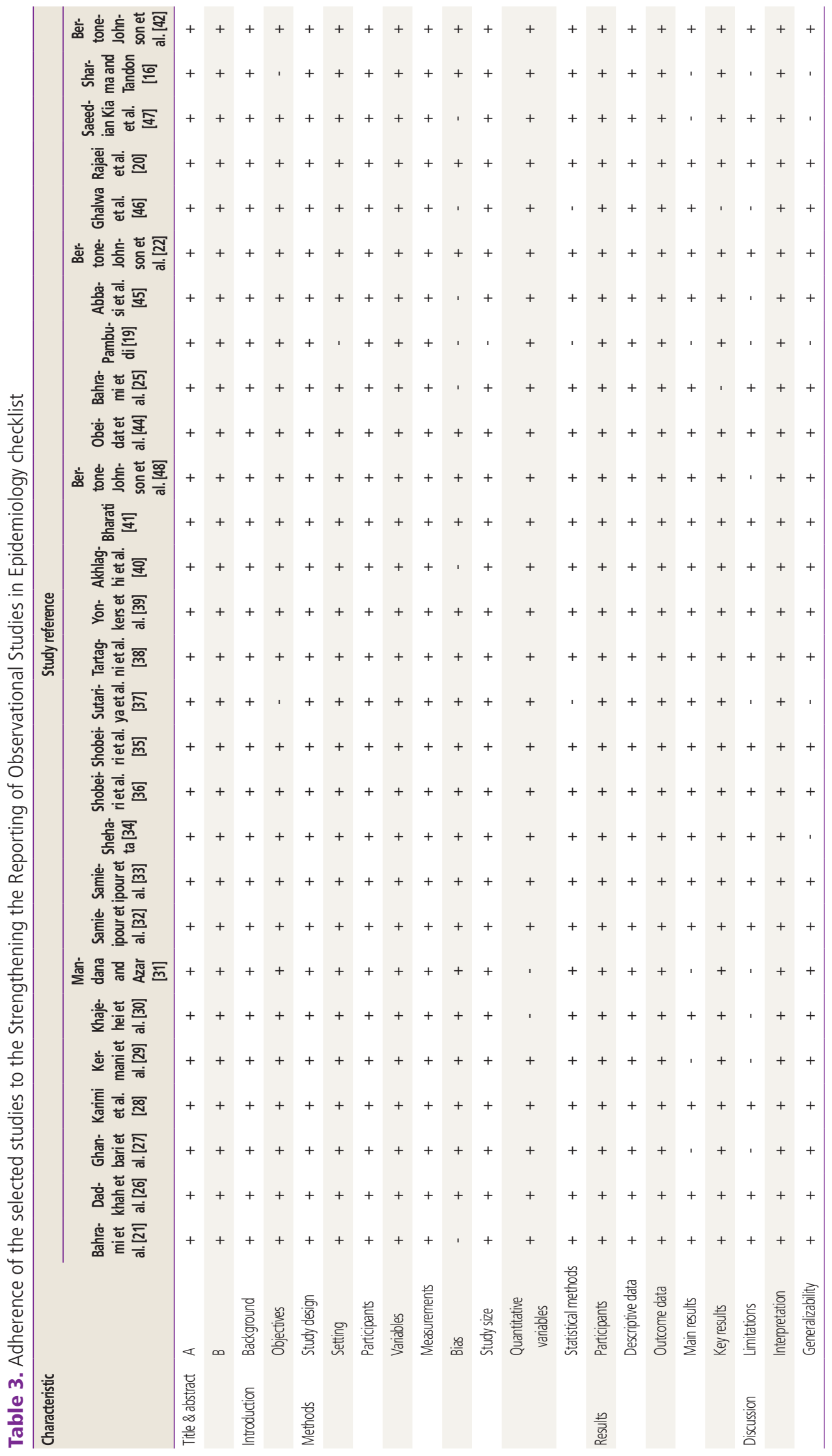




\section{Obstetrics \& Gynecology Science}

Vol. 62, No. 2, 2019

severity $(P<0.05)$. The improvement in symptom ratings was less in this study than in others, but the differences between the scores before and after the intervention were statistically significant.

Bertone-Johnson [56] claimed that low dietary vitamin D was related to the development of PMS. Additionally, high dietary vitamin $D$ intake was suggested to reduce the risk of PMS by affecting calcium levels and oscillation of the cyclic sex steroid hormone, and/or neurotransmitter function $[57,58]$.

Bahrami et al. [21] reported that calcium levels had a significant relationship with the incidence of irritability. Saeedian Kia et al. [47] compared dietary intake and serum levels of calcium, Mg, and vitamin D in students with PMS to those of healthy controls. No significant differences were found between the 2 groups in terms of serum vitamin D levels. The serum concentrations of $\mathrm{Ca}$ and $\mathrm{Mg}$ were lower in some cases, but the levels were within the normal range in all cases.

Bendich [59] showed lower levels of calcium in participants without PMS and concluded that an increase in dietary calcium intake could help maintain normal calcium levels and prevent the manifestation of PMS symptoms. There was a high prevalence of vitamin $D$ deficiency in their population sample of healthy girls. However, the prevalence of PMS symptoms did not differ significantly in relationship to the vitamin D status. Another study in Iran indicated no significant difference in the vitamin D serum status between the PMS and control groups $[21,47]$. In a study on dysmenorrheic college students, Obeidat et al. [44] reported that PMS had no relationship with the levels of parathyroid hormone and vitamin $D$, or intake of dairy products.

Bertone-Johnson et al. [22] performed a nested case-control study and suggested that a high dietary intake of vitamin $D$ and calcium could lower the risk of PMS symptoms. They also observed a significantly lower risk of developing PMS in women with a high intake of vitamin $D$ and calcium from food sources, equivalent to about 4 servings per day of skim or low-fat milk, fortified orange juice, or low-fat dairy foods such as yogurt.

In a clinical trial, Bharati [41] compared yoga exercises and calcium supplements, and observed a considerable reduction in the number of PMS symptoms in the yoga group. Calcium and vitamin D might affect the occurrence of PMS through their associations with endogenous estrogens.

Dadkhah et al. [26] investigated the impacts of vitamins D and $\mathrm{E}$ on PMS. Two months after the intervention, they detected a significant improvement in the PMS symptom scores and reported vitamin $\mathrm{E}$ to be slightly more effective in reducing PMS symptoms than both vitamin $D$ and the placebo. However, there were no significant differences between the 3 groups. Kermani et al. [29] assessed the effects of calcium, vitamin $\mathrm{E}$, and a placebo and reported that, after 3 months of intervention with $1,000 \mathrm{mg}$ of calcium plus $400 \mathrm{IU}$ of vitamin $E$, the severity of symptoms was significantly lower in the intervention group than in the placebo group. In a study by Ghalwa et al. [46], PMS was strongly related with calcium and magnesium. Dietary changes and natural treatments were also linked with the pain and discomfort of women with PMS. PMS also had significant relationships with lifestyle, smoking status, physical activity, and sleep. The effects of dietary behaviors with different types of foods on PMS were also considerable.

Ghanbari et al. [27] performed pre- and post-tests and concluded that depression and anxiety significantly decreased in women treated with calcium compared to the placebo group ( $27 \%$ vs. $7 \% ; P<0.002$ ). Similar results were reported by Bertone-Johnson et al. [22]. Bohrer and Krannich [60] found that calcium supplements effectively improved depressive symptoms.

Karimi et al. [28] investigated the effects of cognitive behavioral therapy (CBT) and calcium plus vitamin D supplementation on the reduction of PMS symptoms. In a trial, Mandana and Azar [31] showed that vitamin E, vitamin B6, calcium, and omega-3 had significant effects on enhancing the physical and mental symptoms of women with PMS. Rajaei et al. [20] observed no relationship between the level of $250 \mathrm{HD}$ during the luteal phase and the presence of PMS. The high prevalence of vitamin D deficiency among the case and control groups might justify this finding. In fact, their study was conducted among young women in Tehran [20], and several studies have indicated a high prevalence of vitamin D deficiency in different parts of Iran [61-63]. Samieipour et al. [32] highlighted significant reductions in the mean total severity of PMS symptoms following both calcium and vitamin B1 supplementation compared to the placebo group $(P<0.001)$. This reduction was more profound in the calcium group. In a randomized and quadruple-blind study, the same authors found calcium plus vitamin B1 supplementation to be more effective on the severity of PMS symptoms than other supplements [33]. 


\section{Obstetrics \& Gynecology Science}

Fatemeh Abdi, et al. The role of calcium and vitamin $D$ in premenstrual syndrome

In a double-blind randomized placebo-controlled trial, Shehata [34] indicated that combined oral contraceptive (COC) pills were superior to calcium in reducing the scores of PMS symptoms. However, the difference was only significant in women who had moderate PMS in the 2 months before treatment, and this can be explained by the mechanisms of action of COC and calcium. If calcium affects PMS through its relationship with endogenous estrogen, it will never be as effective as COCs, which prevent endogenous estrogen more effectively. Another mechanism of COC action is related to neurotransmission.

In a double-blind randomized clinical trial, Shobeiri et al. [35] documented the significant effects of low-dose calcium (500 mg) on the reduction of PMS symptoms. A review study in 2015 evaluated numerous non-pharmacological treatments but only found calcium supplements effective [7]. In a double-blind clinical trial, the same authors observed that calcium intake had significant effects on all physical symptoms (cramps, headache, depression, irritability, general pain, and abdominal pain) of PMS in the first and second months after the intervention [36].

In a prospective, randomized controlled interventional study, Sutariya et al. [37] showed that calcium supplementation effectively mitigated the symptoms of PMS during the luteal phase. The calcium treatment resulted in an approximate $50 \%$ reduction in total mean symptom scores with significant beneficial effects on symptoms including depression, mood swings, headache, irritability, and breast tenderness. Calcium was not effective during the menstrual or intermenstrual phase of the cycle. Tartagni et al. [38] reported that calcium intake, both alone and in combination with vitamin D, was able to reduce the intensity of PMS. In a pilot parallel clinical trial study, Yonkers et al. [39] compared fluoxetine, calcium, and a placebo as a treatment for moderate to severe PMS and PMDD over the course of 4 menstrual cycles. Their findings suggested small benefits of calcium. However, the estimated effect for fluoxetine was much higher than that of calcium. The placebo response in this study (17-33\% depending on the measure) was in line with the range of placebo response rates (11.8-50\%) reported in similar studies [64]. In a cross-sectional study, Bertone-Johnson et al. [42] showed that vitamin D consumption was inversely associated with the prevalence of PMS, and perhaps with menstrual symptom severity in general. In contrast, late luteal phase serum [25(OH)D3] levels were not associated with either out- come.

The review of the selected studies indicates that vitamin $D$ and calcium supplementation, or the use of a diet rich in these two micronutrients, could improve serum levels during the luteal phase, and thus eliminate or improve PMS symptoms.

There has been no unanimously accepted therapeutic method to improve PMS symptoms to date. Vitamin D and calcium, when compared to the treatments mentioned in the studies, such as yoga, CBT, medications like Fluoxetine, COC, etc., can be a safe, effective, acceptable, low cost, and convenient method for reducing the intensity and frequency of PMS symptoms, and thus improving women's quality of life.

One of the limitations of this study was the role of confounding factors, such as age, educational and economic status, geographical area, and mental and physical status of the participants, which were considered in most of the reviewed studies. Since PMS symptoms are recalled by the participants, recall bias was another limitation. Other limitations that were found in some of the studies were lack of control groups, short-term treatments, and low sample size. Moreover, differences in the participants' diets might have affected serum levels of vitamin $D$ and calcium in the studies that did not evaluate and control the women's diet.

\section{Acknowledgements}

This study is related to the project NO 1396/66168 From Student Research Committee, Shahid Beheshti University of Medical Sciences, Tehran, Iran. We also appreciate the "Student Research Committee" and "Research \& Technology Chancellor" in Shahid Beheshti University of Medical Sciences for their financial support of this study.

\section{Conflict of interest}

No potential conflict of interest relevant to this article was reported.

\section{References}

1. Abdnezhad R, Simbar M, Sheikhan Z, Mojab F, Nasiri 


\section{Obstetrics \& Gynecology Science}

Vol. 62, No. 2, 2019

M. The effect of Salvia (Sage) extract on the emotional symptoms of premenstrual syndrome. Iran J Obstet Gynecol Infertil 2017;20:84-94.

2. Brahmbhatt S, Sattigeri BM, Shah H, Kumar A, Parikh D. A prospective survey study on premenstrual syndrome in young and middle aged women with an emphasis on its management. Int J Res Med Sci 2013;1:69-72.

3. Raval CM, Panchal BN, Tiwari DS, Vala AU, Bhatt RB. Prevalence of premenstrual syndrome and premenstrual dysphoric disorder among college students of Bhavnagar, Gujarat. Indian J Psychiatry 2016;58:164-70.

4. Halbreich U, O'Brien PM, Eriksson E, Bäckström T, Yonkers KA, Freeman EW. Are there differential symptom profiles that improve in response to different pharmacological treatments of premenstrual syndrome/premenstrual dysphoric disorder? CNS Drugs 2006;20:523-47.

5. Kahyaoglu Sut $H$, Mestogullari E. Effect of premenstrual syndrome on work-related quality of life in Turkish nurses. Saf Health Work 2016;7:78-82.

6. Sepehrirad M, Bahrami H, Noras M. The role of complementary medicine in control of premenstrual syndrome evidence based (Regular Review Study). Iran J Obstet Gynecol Infertil 2016;19:11-22.

7. Freeman EW. Therapeutic management of premenstrual syndrome. Expert Opin Pharmacother 2010;11:2879-89.

8. World Health Organization. National policy on traditional medicine and regulation of herbal medicines: report of a WHO global survey. Geneva: World Health Organization; 2005.

9. Babazadeh R, Keramat A. Premenstrual syndrome and complementary medicine in Iran: a systematic review. Feyz J Kashan Univ Med Sci 2011;15:174-87.

10. Mun MJ, Kim TH, Hwang JY, Jang WC. Vitamin D receptor gene polymorphisms and the risk for female reproductive cancers: a meta-analysis. Maturitas 2015;81:256-65.

11. Kim TH, Lee HH, Kim JM, Lee A, Park J, Kim Y. A comparison in vitamin $D$ receptor expression during oral menopausal hormone therapy and vaginal estrogen therapy. Clin Exp Obstet Gynecol 2018;45:39-43.

12. Cioni F, Ferraroni F. Vitamin $D$ and other nutrients in the treatment of premenstrual syndrome. In: Hollins-Martin C, van den Akker O, Martin C, Preedy VR, editors. Handbook of diet and nutrition in the menstrual cycle, periconception and fertility. Wageningen: Wageningen
Academic Publishers; 2014. p.121-36.

13. Lee $A$, Lee MR, Lee HH, Kim YS, Kim JM, Enkhbold T, et al. Vitamin $D$ proliferates vaginal epithelium through RhoA expression in postmenopausal atrophic vagina tissue. Mol Cells 2017;40:677-84.

14. Taghizadeh Z, Shirmohammadi M, Feizi A, Arbabi M. The effect of cognitive behavioural psycho-education on premenstrual syndrome and related symptoms. J Psychiatr Ment Health Nurs 2013;20:705-13.

15. Sen AP, Gulati $A$. Use of magnesium in traumatic brain injury. Neurotherapeutics 2010;7:91-9.

16. Sharma G, Tandon P. Luteal phase serum calcium and serum magnesium levels in causation of premenstrual syndrome. Int J Basic Appl Physiol 2015;4:126-30.

17. Vaidya A, Forman JP. Vitamin D and vascular disease: the current and future status of vitamin $D$ therapy in hypertension and kidney disease. Curr Hypertens Rep 2012;14:111-9.

18. Forman JP, Williams JS, Fisher ND. Plasma 25-hydroxyvitamin $D$ and regulation of the renin-angiotensin system in humans. Hypertension 2010;55:1283-8.

19. Pambudi MF. Calcium level is lower in women with premenstrual syndrome. Majalah Obstet Ginekol Indones 2013;37:99-102.

20. Rajaei S, Akbari Sene A, Norouzi S, Berangi Y, Arabian S, Lak $P$, et al. The relationship between serum vitamin $D$ level and premenstrual syndrome in Iranian women. Int J Reprod Biomed (Yazd) 2016;14:665-8.

21. Bahrami A, Bahrami-Taghanaki H, Afkhamizadeh M, Avan A, Mazloum Khorasani Z, Esmaeili $H$, et al. Menstrual disorders and premenstrual symptoms in adolescents: prevalence and relationship to serum calcium and vitamin D concentrations. J Obstet Gynaecol 2018;38:989-95.

22. Bertone-Johnson ER, Hankinson SE, Bendich A, Johnson SR, Willett WC, Manson JE. Calcium and vitamin D intake and risk of incident premenstrual syndrome. Arch Intern Med 2005;165:1246-52.

23. Lo CK, Mertz D, Loeb M. Newcastle-Ottawa Scale: comparing reviewers' to authors' assessments. BMC Med Res Methodol 2014;14:45.

24. Roozbeh N, Banihashemi F, Mehraban M, Abdi F. Potential role of Factor $\mathrm{V}$ Leiden mutation in adverse pregnancy outcomes: an updated systematic review. Biomed Res Ther 2017;4:1832-46. 


\section{Obstetrics \& Gynecology Science}

Fatemeh Abdi, et al. The role of calcium and vitamin $\mathrm{D}$ in premenstrual syndrome

25. Bahrami A, Avan A, Sadeghnia HR, Esmaeili H, Tayefi M, Ghasemi $F$, et al. High dose vitamin D supplementation can improve menstrual problems, dysmenorrhea, and premenstrual syndrome in adolescents. Gynecol Endocrinol 2018;34:659-63.

26. Dadkhah H, Ebrahimi E, Fathizadeh N. Evaluating the effects of vitamin $D$ and vitamin $E$ supplement on premenstrual syndrome: a randomized, double-blind, controlled trial. Iran J Nurs Midwifery Res 2016;21:159-64.

27. Ghanbari Z, Haghollahi F, Shariat M, Foroshani AR, Ashrafi M. Effects of calcium supplement therapy in women with premenstrual syndrome. Taiwan J Obstet Gynecol 2009;48:124-9.

28. Karimi Z, Dehkordi MA, Alipour A, Mohtashami T. Treatment of premenstrual syndrome: appraising the effectiveness of cognitive behavioral therapy in addition to calcium supplement plus vitamin D. PsyCh J 2018;7:4150.

29. Kermani AZ, Taavoni S, Hosseini AF. Effect of combined calcium and vitamin $\mathrm{E}$ consumption on premenstrual syndrome. Iran J Nurs 2010;23:8-14.

30. Khajehei M, Abdali K, Parsanezhad ME, Tabatabaee HR. Effect of treatment with dydrogesterone or calcium plus vitamin $D$ on the severity of premenstrual syndrome. Int J Gynaecol Obstet 2009;105:158-61.

31. Mandana Z, Azar A. Comparison of the effect of vit $E$, vitB6, calcium and omega-3 on the treatment of premenstrual syndrome: a clinical randomized trial. Annu Res Rev Biol 2014;4:1141-9.

32. Samieipour S, Kiani F, Samiei Pour Y, Babaei Heydarabadi A, Tavassoli E, Rahim Zade R. Comparing the effects of vitamin $B 1$ and calcium on premenstrual syndrome (PMS) among female students, Ilam-Iran. Int J Pediatr 2016;4:3519-28.

33. Samieipour S, Tavassoli E, Heydarabadi B, Daniali SS, Alidosti $M$, Kiani $F$, et al. Effect of calcium and vitamin B1 on the severity of premenstrual syndrome: a randomized control trial. Int J Pharm Technol 2016;8:18706-17.

34. Shehata NA. Calcium versus oral contraceptive pills containing drospirenone for the treatment of mild to moderate premenstrual syndrome: a double blind randomized placebo controlled trial. Eur J Obstet Gynecol Reprod Biol 2016;198:100-4.

35. Shobeiri F, Araste FE, Ebrahimi R, Jenabi E, Nazari M. Effect of calcium on premenstrual syndrome: a double- blind randomized clinical trial. Obstet Gynecol Sci 2017;60:100-5.

36. Shobeiri F, Ezzati Arasteh F, Ebrahimi R, Nazari M. Effect of calcium on physical symptoms of premenstrual syndrome. Iran J Obstet Gynecol Infertil 2016;19:1-8.

37. Sutariya S, Talsania N, Shah C, Patel M. An interventional study (calcium supplementation \& health education) on premenstrual syndrome - effect on premenstrual and menstrual symptoms. Natl J Community Med 2011;2:100-4.

38. Tartagni M, Cicinelli MV, Tartagni MV, Alrasheed H, Matteo $M$, Baldini D, et al. Vitamin D supplementation for premenstrual syndrome-related mood disorders in adolescents with severe hypovitaminosis D. J Pediatr Adolesc Gynecol 2016;29:357-61.

39. Yonkers KA, Pearlstein TB, Gotman N. A pilot study to compare fluoxetine, calcium, and placebo in the treatment of premenstrual syndrome. J Clin Psychopharmacol 2013;33:614-20.

40. Akhlaghi F, Hamedi A, Javadi Z, Hosseinipoor F. Effects of calcium supplementation on premenstrual syndrome. Razi J Med Sci 2004;10:669-75.

41. Bharati M. Comparing the effects of yoga \& oral calcium administration in alleviating symptoms of premenstrual syndrome in medical undergraduates. J Caring Sci 2016;5:179-85.

42. Bertone-Johnson ER, Chocano-Bedoya PO, Zagarins SE, Micka $A E$, Ronnenberg AG. Dietary vitamin D intake, 25-hydroxyvitamin D3 levels and premenstrual syndrome in a college-aged population. J Steroid Biochem Mol Biol 2010;121:434-7.

43. Mortola JF, Girton L, Beck L, Yen SS. Diagnosis of premenstrual syndrome by a simple, prospective, and reliable instrument: the calendar of premenstrual experiences.Obstet Gynecol 1990;76:302-7.

44. Obeidat BA, Alchalabi HA, Abdul-Razzak KK, Al-Farras $\mathrm{MI}$. Premenstrual symptoms in dysmenorrheic college students: prevalence and relation to vitamin $D$ and parathyroid hormone levels.Int J Environ Res Public Health 2012;9:4210-22.

45. Abbasi ST, Abbasi P, Suhag AH, Qureshi MA. Serum magnesium and 25- hydroxy cholecalciferol in premenstrual syndrome during luteal phase. J Liaquat Uni Med Health Sci 2017;16:209-12.

46. Ghalwa NA, Qedra R, Wahedy K. Impact of calcium 


\section{Obstetrics \& Gynecology Science}

Vol. 62, No. 2, 2019

and magnesium dietary changes on women pain and discomfort from premenstrual syndrome at the Faculty of Pharmacy-Gaza strip. World J Pharm Pharm Sci 2014;3:981-1005.

47. Saeedian Kia A, Amani R, Cheraghian B. The association between the risk of premenstrual syndrome and vitamin D, calcium, and magnesium status among university students: a case control study. Health Promot Perspect 2015;5:225-30.

48. Bertone-Johnson ER, Hankinson SE, Forger NG, Powers SI, Willett WC, Johnson SR, et al. Plasma 25-hydroxyvitamin $D$ and risk of premenstrual syndrome in a prospective cohort study. BMC Womens Health 2014;14:56.

49. American College of Obstetricians and Gynecologists. Frequently asked questions FAQ057: gynecologic problems: premenstrual syndrome. Washington, D.C.: American College of Obstetricians and Gynecologists; 2011.

50. Thys-Jacobs S, McMahon D, Bilezikian JP. Cyclical changes in calcium metabolism across the menstrual cycle in women with premenstrual dysphoric disorder. J Clin Endocrinol Metab 2007;92:2952-9.

51. Eyles DW, Burne TH, McGrath JJ. Vitamin D, effects on brain development, adult brain function and the links between low levels of vitamin $D$ and neuropsychiatric disease. Front Neuroendocrinol 2013;34:47-64.

52. Skowrońska P, Pastuszek E, Kuczyński W, Jaszczoł M, Kuć $P$, Jakiel $G$, et al. The role of vitamin $D$ in reproductive dysfunction in women - a systematic review. Ann Agric Environ Med 2016;23:671-6.

53. Miyashita M, Koga K, Izumi G, Sue F, Makabe T, Taguchi $A$, et al. Effects of 1, 25-dihydroxy vitamin D3 on endometriosis. J Clin Endocrinol Metab 2016;101:2371-9.

54. Harris HR, Chavarro JE, Malspeis S, Willett WC, Missmer SA. Dairy-food, calcium, magnesium, and vitamin D intake and endometriosis: a prospective cohort study. Am
J Epidemiol 2013;177:420-30.

55. Holick MF, Binkley NC, Bischoff-Ferrari HA, Gordon CM, Hanley DA, Heaney RP, et al. Guidelines for preventing and treating vitamin $D$ deficiency and insufficiency revisited. J Clin Endocrinol Metab 2012;97:1153-8.

56. Bertone-Johnson ER. Vitamin D and the occurrence of depression: causal association or circumstantial evidence? Nutr Rev 2009;67:481-92.

57. Holick MF. Vitamin D: a D-Lightful health perspective. Nutr Rev 2008;66:S182-94.

58. Thys-Jacobs S. Micronutrients and the premenstrual syndrome: the case for calcium. J Am Coll Nutr 2000;19:220-7.

59. Bendich A. The potential for dietary supplements to reduce premenstrual syndrome (PMS) symptoms. J Am Coll Nutr 2000;19:3-12.

60. Bohrer T, Krannich JH. Depression as a manifestation of latent chronic hypoparathyroidism. World J Biol Psychiatry 2007;8:56-9.

61. Faghih S, Abdolahzadeh M, Mohammadi M, Hasanzadeh J. Prevalence of vitamin d deficiency and its related factors among university students in Shiraz, Iran. Int J Prev Med 2014;5:796-9.

62. Kaykhaei MA, Hashemi M, Narouie B, Shikhzadeh A, Rashidi H, Moulaei N, et al. High prevalence of vitamin D deficiency in Zahedan, southeast Iran. Ann Nutr Metab 2011;58:37-41.

63. Hovsepian S, Amini M, Aminorroaya A, Amini P, Iraj $B$. Prevalence of vitamin $D$ deficiency among adult population of Isfahan City, Iran. J Health Popul Nutr 2011;29:149-55.

64. Halbreich U. Selective serotonin reuptake inhibitors and initial oral contraceptives for the treatment of PMDD: effective but not enough. CNS Spectr 2008;13:566-72. 